\title{
Micrometer-long covalent organic fibres by photo-initiated chain-growth radical polymerization on an alkali-halide surface
}

\author{
Franck Para ${ }^{1}$, Franck Bocquet ${ }^{1}$, Laurent Nony ${ }^{1}$, and Christian Loppacher ${ }^{1 *}$; \\ Michel Féron ${ }^{2}$ and Fréderic Cherioux ${ }^{2}$; \\ David Z. Gao ${ }^{3}$, Filippo Federici Canova ${ }^{3}$, and Matthew B. Watkins ${ }^{4}$. \\ ${ }_{1}^{1}$ Aix-Marseille Université, CNRS, IM2NP UMR 7334, 13397 Marseille, France; \\ 2 Institut FEMTO-ST, Univ. Bourgogne Franche-Comté, CNRS, 15B avenue des Montboucons, \\ F-25030 Besançon, France; \\ ${ }^{3}$ Nanolayers Research Computing LTD, 15 Southgrove Road, Sheffield, UK, S10 2NP; \\ ${ }^{4}$ School of Mathematics and Physics, University of Lincoln, UK.
}

\begin{abstract}
:
On-surface polymerization is a promising technique to prepare organic functional nanomaterials that are challenging to synthesize in solution, but it is typically used on metal substrates, which play a catalytic role. Previous examples on insulating surfaces have involved intermediate selfassembled structures, which face high barriers to diffusion, or annealing to higher temperatures, which causes rapid dewetting and desorption of the monomers. Here we report the photoinitiated radical polymerization, initiated from a two-dimensional gas phase, of a dimaleimide monomer on an insulating $\mathrm{KCl}$ surface. Polymer fibres up to $1 \mu \mathrm{m}$ long are formed through chain-like rather than step-like growth. Interactions between potassium cations and the dimaleimide's oxygen atoms facilitate propagation of the polymer fibres along a preferred axis of the substrate over long distances. Density functional theory calculations, noncontact atomic force microscopy imaging, and manipulation at room temperature were used to explore the initiation and propagation processes, as well as the structure and stability of the resulting 1D polymer fibres.
\end{abstract}




\section{Main Text:}

On-surface polymerization of organic precursors provides new possibilities to form highly-stable and atomically-defined nanostructures that exhibit desired electronic, optical, catalytic etc. properties. Compared to standard chemistry using isotropic solution in a test tube, on-surface reactions are confined to two dimensions (2D). This spatial confinement of the reaction gives access to entirely new reaction pathways due to for example the stabilizing of specific intermediate species. Furthermore, the substrate for on-surface reactions can be chosen in order to control both a catalytic as well as a templating effect to guide the size, the shape, and the nature of the structures formed. As emphasized by the Nobel Prize in Physics in 2016, ${ }^{1,2}$ awarded for early work in understanding topological phase transitions and topological phases of matter, the formation of artificial matter exhibiting properties controlled by their symmetry is very promising in nanoelectronics. ${ }^{3,4}$ This issue can be addressed by creating artificial 1D polymers with innovative properties, for example by polymerization of "exotic" polymorphs only accessible on insulating surfaces. The state of the art for on-surface synthesized, highly ordered 1D or 2D polymers are dimensions of about $100 \mathrm{~nm} .{ }^{5,6}$ For the basic building blocks of molecular circuitry to interconnect active devices, there is however a need to fabricate isolated nanowires with a length larger than $1 \mu \mathrm{m}$. This is still a challenge due to the fact that top-down approaches such as photolithography or etching fail to achieve the formation of isolated, long and mechanically stable nanowires.

In the last years, special interest was given to on-surface reactions performed under ultra-high vacuum (UHV) conditions and on atomically clean single-crystal metal substrates. In this solvent-free environment, classical chemical reactions such as Ullmann type coupling, 7, 8, 9, 10, 11, ${ }^{12}$ Glaser coupling, ${ }^{13,14}$ imine formation, ${ }^{15}$ self-condensation of boronic acids, ${ }^{16,17,18}$ and many more (for overviews see refs. ${ }^{19,20,6,21,22,23}$ ) have successfully been used to create well-defined and covalently bound organic $1 \mathrm{D}$ and $2 \mathrm{D}$ structures. Key factors for this success are first the confinement to 2D and the adsorption on a well-chosen surface that can favour both thermodynamic and kinetic progression of the polymerization process. ${ }^{24}$ Second, in UHV there is a much broader range of annealing temperatures possible. In fact, most of the reactions described so far are initiated by thermal annealing which however often creates defects in the formed structures due to the increased diffusion of the precursors on the surface. In order to circumvent this problem, some work was reported where the polymerization was initiated by UVillumination ${ }^{12,25,26,27,28}$ or current injection by means of an scanning tunnelling microscopy tip. ${ }^{7}$, 29, 30,31 The main limitations of the so far created structures in view of future applications in nanoscale electronic and optical devices are (i) the use of metal substrates (for instance, leading to non-radiative quenching ${ }^{32}$ ), (ii) the high number of defects in the formed covalent structures, and (iii) the side-products of some reactions which might remain on the substrate surface.

(i) The limitations linked to the use of metal substrates with their catalytic effect ${ }^{33,34,26}$ have been circumvented by applying on-surface reactions by thermal annealing on thin decouplinglayers. ${ }^{35,36,37,38}$ The attempts to use ultrathin spacer layers are encouraging, however, the initiation of the used reactions still needed the presence of the metal surface. ${ }^{35,36}$ Furthermore, it was shown that for electronic devices with predominant lateral transport the spacer layers must have a thickness of at least 3-4 monolayers (ML) $)^{35}$ and when permanent charging of single 
molecules is desired, the layer must be even thicker. ${ }^{39}$ On bulk insulating substrates, successful polymerization was so far obtained on calcite ${ }^{40,41,42,43,44}$ and mica ${ }^{45}$ surfaces which have a high surface energy ${ }^{46}$ and provide a strong anchoring of the molecules to the substrates which allows for relatively large annealing temperatures (500K). ${ }^{42}$ UV-induced polymerization on bulk insulators was demonstrated for pre-arranged supramolecular structures for both $1 \mathrm{D}^{44}$ and $2 \mathrm{D}$ structures. ${ }^{42}$

(ii) The so far largest, defect-free organic layers have been achieved by supramolecular selfassembly (SA) in which the molecule-molecule (MM) interaction is dominated by relatively weak and reversible Van der Waals (VdW) or Hydrogen bonding. In such systems, the molecular layer can achieve its highly-ordered thermodynamic equilibrium, however, the technological application of weakly bound organic layers is very limited due to their poor thermal and chemical stability. Several attempts have been made to increase the structural order of covalent organic networks by either using supramolecular SA as intermediate step before covalent linking was initiated $^{26,34,47,48,49,50,51}$ or by performing covalent linking under aqueous solution where the polymerization becomes reversible. ${ }^{52}$

(iii) Side products of the polymer reaction can have two negative effects: namely surface poisoning by for example halogen atoms ${ }^{12}$ or residuals which sterically hinder the defect-free growth. These problems can either be circumvented by using side product-free reactions or by choosing reaction temperatures at which the side products desorb from the substrate surface.

Here, we propose a technique that can overcome the three obstacles identified above by using a side product-free 1D polymerization on an alkali-halide surface. This concept is based on light-induced radical polymerization, ${ }^{53}$ which is a powerful method to obtain composite materials and polymers at the industrial scale, but which has only rarely been addressed on conducting surfaces $^{54,55,56,57,58}$ and has never been transferred on the surface of bulk insulators so far, despite of its efficiency in the absence of any catalytic role of the surface. The highly ordered and long nanofibres are formed owing to: the fact that the UV-induced radical polymerization is chain-like, that it is guided by the molecule-substrate interaction, and that the reaction takes place in a diluted 2D gas phase acting as a reservoir of the precursor molecules that allows unhindered propagation, rather than through pre-arranged structures, as has previously been shown on insulating surfaces. ${ }^{40-44}$ In addition, photo-induced polymerization does not require thermal annealing (by opposition with catalytic on-surfaces reactions) which is a major advantage in order to avoid the formation of defects during the polymerisation. The conclusions on the polymerization process are supported by Density Functional Theory (DFT) and Climbing Image Nudged Elastic Band (NEB) calculations, and the structure and stability of the covalent micrometer-long organic fibres are verified experimentally by noncontact Atomic Force Microscopy (ncAFM).

\section{Results}

Aiming to form fibres that may find practical use in molecular devices, we looked to develop an efficient and robust synthesis method for well-oriented micrometre-long nanowires on the surface of bulk insulators. We chose the family of maleimide molecules since they are widely 
used in the synthesis of high performance macromolecular systems. ${ }^{59,60}$ Furthermore, they can participate in polymerizations that occur through nucleophilic reactions, cycloaddition and radical polymerization. ${ }^{61,62}$ Different from step-growth polymerization mechanisms (such as Ullman cross-coupling), radical polymerization is not controlled by the diffusion of species that have previously been activated. Thus, in radical polymerization, the size of the polymers formed does not significantly depend on the reaction time as is the case for step-growth reaction where a very long reaction time is required to obtain highly-ordered and large-scale polymers. ${ }^{63}$ With these considerations in mind, we turned to radical polymerization on surfaces. The fact that first, their radical polymerization can be initiated by UV-light even in the absence of a photoinitiator, ${ }^{64}$ and second, that it does not require an intermediate self-assembled organization of the molecules on the substrate, makes maleimides ideal building blocks to obtain micrometre-sized nanofibres on insulating surfaces in UHV.

We used N,N'-(1,4-Phenylene)dimaleimide as building blocks due to the presence of two maleimide rings which should facilitate the growth of the targeted nanofibres. In addition, the presence of electron-rich $\mathrm{C}=\mathrm{O}$ favours the electrostatic molecule-substrate interaction between the four oxygen atoms of the molecule and alkali cations of the surface. ${ }^{65}$ The MS interactions have been investigated by DFT calculations on the $\mathrm{KCl}(001)$ surface. The binding energy of 1.4 $\mathrm{eV}$ per molecule is to a large extent caused by VdW interactions $(0.9 \mathrm{eV})$. The strongest sitespecific interaction is formed between the oxygen atoms of the two maleimide moieties pointing towards $\mathrm{K}^{+}$cations of the surface. Charge density difference plots show weak polarizations stabilizing mainly ionic interactions between these oxygen atoms and $\mathrm{K}^{+}$cations (Figure 1), as well as polarization of aromatic ring density towards surface cations. The configuration presented in Figure 1 is the energetically most favoured, however, other configurations with the central benzene ring parallel to the surface and the maleimide rings turned out of plane (and thus only one oxygen atom per maleimide ring interacting with a surface cation) are energetically very close. Therefore, especially at room temperature (RT), monomers diffusing on the surface can find their position to undergo a covalent bond to neighbouring (radical) molecules.

\section{Surface-induced 1D Polymer on KCl (without UV illumination)}

Figure 2a-c display ncAFM images of the polymer fibres formed when $0.7 \mathrm{ML}$ of dimaleimide derivatives were deposited onto the $\mathrm{KCl}$ (001) surface. Upon deposition, the molecules form some diffusing 2D self-assembled structures and a diluted gas phase which slowly (in a few hours) dewet to form large 3D crystallites (see Supplementary Figure 4). As long as the monomers diffuse on the surface the polymerization continues, and fibres of up to $1 \mu \mathrm{m}$ length were formed. The fibres are oriented along the $<100\rangle$ and $<010\rangle$ directions of the $\mathrm{KCl}$ substrate (indicated by $a_{K C L}$ and $b_{K C l}$ in the figures). In areas where the fibres are well aligned with the substrate and stabilized (by clusters or other fibres), the inner structure shows a slight rumpling which is evidenced in a zig-zag structure of alternating dots showing a periodicity of $25 \AA \pm 1 \AA$ (Figure 2b). A detailed analysis of the inner fibre structure as shown in Figure 2c permits to establish a geometrical model of the structure. The experimentally observed zig-zag structure 
contains two times five molecules per dot, oriented oppositely and polymerized in a "zip-like" geometry. The mean separation between molecules along a chain is $5 \AA \pm 0.2 \AA$ which can be deduced either from the periodicity of the zig-zag structure in Figure $2 \mathrm{~b}$ or from an evaluation of Figure $2 \mathrm{c}$ taking into account the simultaneously resolved $\mathrm{KCl}$ substrate with its known lattice constant (centred unit cell of $6.3 \AA$, only one type of ion is visible in ncAFM images). The observed zig-zag structure comes from the fact that the maleimide molecules along the fibre are positioned above potassium cations along the $\langle 100\rangle$ or $<010\rangle$ direction of the $\mathrm{KCl}$ substrate. The fact that the positions of these species coincide with each other is evidenced geometrically by the agreement of five molecular distances $5^{\star} 5 \AA \approx 25 \AA$ with four $\mathrm{KCl}$ unit cells $4^{*} 6.3 \AA=25.2 \AA$. Figure $2 d$ displays a structure obtained by DFT/MD (Molecular Dynamics) calculations which take as input the experimentally deduced geometrical model (detailed description of the DFT/MD calculations below). The central polymer carbon chain is $s p^{3}$ hybridized and non-planar.

\section{Effect of UV-illumination}

Figure 3 shows the comparison of a sample prepared without and one with UV-illumination by a light emitting diode (LED, 14 hours illumination). The fibres on the illuminated sample are interlinked with each other and form a network which in some areas encloses remaining 2D supramolecular arrangement (which can no longer diffuse and dewet into 3D crystallites). Due to the relatively small intensity of the UV light, the initiation of the chain-like polymerization is still a rare event, however about two orders of magnitude more frequent than without UV light as can be seen by the number of fibres formed in Figure 3b compared to Figure 3a. On illuminated sample areas, the propagation of the chain polymerization is limited by neighbouring fibres.

\section{Stability of the 1D polymer fibres}

Different ways are described in literature in order to prove that an observed structure is really covalently bound: most frequently geometrical comparison with calculated structures or thermal stability are evaluated, but also manipulation by an STM tip were used. ${ }^{7,10,35,49,66}$ Besides the above described structural calculations (Figure 2d) which are in excellent agreement with the experimental images, and an evaluation of the thermal stability (Supplementary Figure 3) which confirms the covalent linking of the molecules, we also tested the mechanical stability of the fibres. Thereto, the ncAFM tip was placed on top of a chosen fibre and approached until an instability occurred. Figure 4 shows the images of pairwise identical sample areas before and after a mechanical manipulation at the point indicated with a black cross in figs. a, c, and e. The fibre in the first manipulation (Figure $4 a$ and b) detaches from the step edge in the lower part of the image and changes its conformation upon the indentation of the tip in the upper part. The fibre in the second series (figs. $c$ and $d$ ) is completely removed from the surface (presumably onto the tip), and in the third series (figs. e and f) the already bent fibre completely changes its form. These three examples clearly prove the mechanical stability of the fabricated, covalentlybound fibres. 


\section{Discussion}

The $N, N^{\prime}$-(1,4-Phenylene)dimaleimide molecules adsorb in a rather flexible geometry on the $\mathrm{KCl}$ surface with always at least two or even four oxygen atoms interacting with the $\mathrm{K}^{+}$cations of the surface (see Figure 1). The dimaleimide monomers diffuse on the surface at RT and form a 2D gas phase before they slowly dewet to form large 3D crystals (for more details about the nucleation and growth see Supplementary Figures 4 and 5). The polymerization is most probably initiated by the flexible conformation of the molecules on the surface which allows for a rotation of the maleimide rings and thus facilitates the $\mathrm{C}-\mathrm{C}$ bond formation between neighbouring molecules. The polymerization starts by electrophilic addition of another molecule, leading to the polymer formation (see Supplementary Figure 1). This process is still rare and poorly effective because $\mathrm{K}^{+}$cations are not as strong electrophiles as usually used for maleimide polymerization. ${ }^{59}$ Therefore, only a few nanofibres are formed in the absence of UVlight (Figure 3a).

In the case of UV-light illumination, the process of polymerization is well known in solution or in solid state. The initiation step is based on the formation of a triplet biradical species due to the absorption of light. Then the propagation step is based on the addition of other maleimide molecules to this biradical species (Supplementary Figure 2). The formation of generated triplet biradical species on the $\mathrm{KCl}$ surface has been investigated by means of DFT simulations. The formation of a triplet biradical of $N^{\prime} N^{\prime}-(1,4-$ Phenylene)dimaleimide molecule adsorbed on $\mathrm{KCl}$ requires $2.1 \mathrm{eV}$. Therefore, the UV-light excitation of $N, N^{\prime}-(1,4-$ Phenylene)dimaleimide molecules is efficient and the growth of nanowires is initiated (Figure $3 b$ ). The addition of a second $N, N^{\prime}-(1,4-$ Phenylene)dimaleimide strongly stabilizes the corresponding triplet biradical due to the formation of a $\mathrm{C}-\mathrm{C}$ bond, its energy being $0.40 \mathrm{eV}$ below that of the adsorbed reactants. Once the growth is initiated by the formation of a biradical dimer it is only stopped by either surface defects (clusters, neighbouring fibres) or by exhaustion of the precursor molecules due to dewetting for example. Please note that by a proper choice of the molecule's polar end-groups and the used alkali-halide substrate, both the diffusion and the dewetting can be controlled precisely as we have shown for other molecules. ${ }^{65}$

In order to gain more insight into the reaction mechanisms involved in the nucleation of the polymer chains we calculated the energies of the transition states (TS) by means of the NEB method. These calculations confirm that the nucleation via a biradical dimer and the subsequent propagation are possible by a single molecule in the excited state (Supplementary Figure 6). The localised nature of the biradical species dictates the growth of the chain (Supplementary Figure 7): the radical sites are the only locations a monomer can add to the growing chain.

Finally, we simulated a polymer of $N^{\prime} N^{\prime}-(1,4-$ Phenylene)dimaleimide, with repeat unit of 5 molecules, along the $<100>$ or $<010>$ direction of the $\mathrm{KCl}$ surface by DFT/MD calculations. The binding energy in this infinite fibre increases to $1.9 \mathrm{eV}$ per molecule, compared to the $1.4 \mathrm{eV}$ for a single $N, N^{\prime}-(1,4-$ Phenylene)dimaleimide on $\mathrm{KCl}$. This larger binding energy mainly originates from intrachain $\mathrm{VdW}$ interactions. The electrostatic interactions between oxygen atoms of the 
outer maleimides ring (which were not involved in the polymerization process) with $\mathrm{K}^{+}$cations determine the preferred adsorption geometry.

The observed polymerization is a chain-growth reaction in which the reactive species (that is, the growing fibres) do not need to diffuse to react. The fact that we observe the formation of large and probably defect-free fibres is largely due to the combination of a 2D gas phase that provides sufficient precursor molecules with a radical polymerisation mechanism in which the structure grows molecule by molecule to reach lengths of up to one micrometre.An unhindered propagation of the growth is only possible in a 2D gas (once initiated); in contrast, in cases involving a 2D self-assembled intermediate structure, the growth would quickly be stopped as no precursor molecules could diffuse to increase the fibre length. The only exception in which a regular self-assembled structure could be polymerised into a defect-free covalent one would be when the lattices of both structures are identical. Additionally, we feel that the substrate templating effect also contributes to the formation of fibres that are mostly defect-free. The above described binding energy between oxygen atoms of the growing fibre and the cations of the substrate stabilises the fibre and guides the formation in the $<100>$ or $<010>$ direction of the ionic surface.

In a step-growth reaction, dimers and multimers would be formed all over the surface. Due to the increasing binding energy of these aggregates (that is, monomers that form oligomers), larger aggregates would no longer be able to diffuse on the substrate and optimize their geometry in order to form a long, regular structure.

We believe that the formed fibres are defect-free although we were not able to image the full length of a fibre with molecular resolution. We feel that this conclusion is supported by (i) the mechanism of radical polymerization, where one initiation leads to a very long polymer fibre which grows rather fast (Supplementary Figure 5). Our calculations of the radical spin density show that there is only one exact position on each fibre end where the polymerization can proceed. This indicates that, at least for 1D chain growth, it is not probable that a second initiation would happen exactly at the place where an existing fibre was terminated, causing a defect in the resulting fibre. (ii) The fact that the observed zig-zag structure is caused by oxygen groups of the monomers in the fibre being located above potassium cations on the surface. The zig-zag structure observed is very regular, suggesting that it is defect-free. (iii) Finally, the shapes of some fibres deviates from perfect linearity. In our opinion, this comes from weak interactions between the fibres and the substrate, and the fact that the fibres can easily be bent. Therefore, any defects in or on the surface can easily disturb the linear appearance of the fibres. This was more frequently observed on surfaces with more fibres than the ones in Figure 2 , where most fibres appear regular and aligned with the substrate crystallographic axis.

\section{Conclusion}

We present a radical, side-product-free polymerization on a bulk insulating surface that leads to the formation of micrometer-long polymer fibres. In this approach, first, the molecule and alkalihalide surface chosen enable the deposited precursor molecules to form a 2D gas phase rather than a rigid self-assembled layer. This in turn leads to the formation of long and regular 
structures, as the precursor molecules are easily accessible, but the radicals do not need to diffuse or rearrange to form the covalent structure. Second, the molecule-substrate electrostatic interaction and the molecule's conformation on the substrate allow for both rare spontaneous initiation and UV-light induced initiation of the radical polymerization at room temperature. Once initiated, the chain-like polymerization proceeds easily and is only stopped by either defects on the surface or exhaustion of the precursor molecules. In future work, we will focus on applying this synthetic method to the formation of two-dimensional and electrically conducting structures.

\section{Methods}

Design of maleimide molecules

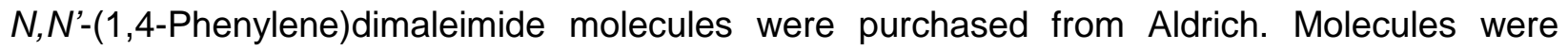
purified by recrystallization in propan-2-ol prior to use.

\section{Sample preparation}

Ionic single-crystal substrates (MaTeck Material-Technologie \& Kristalle $\mathrm{GmbH}$, Im Langenbroich 20, 52428 Jülich, Germany) were cleaved ex situ, quickly introduced into UHV and annealed for one hour to $240^{\circ} \mathrm{C}$ in order to achieve clean surfaces presenting large terraces and evaporation spirals. Approximately 0.7 monolayer (ML) of dimaleimide molecules were deposited from a home-made evaporation cell using quartz crucibles (evaporation temperature $115^{\circ} \mathrm{C}$ ) onto the sample kept at room temperature. Evaporation rates were set to about 0.5 monolayer per minute by monitoring the deposition by means of a quartz oscillator and a frequency meter (TF930 from Aim-TTi: Glebe Road, Huntingdon, Cambridgeshire, PE29 7DR United Kingdom). Some samples were irradiated for half a day by an UV LED kept ex situ (Roithner DUV265-HL5NR, 265nm (4.7eV), 0.5mW, TO5 case with internal reflector and hemispherical quartz glass lens; ROITHNER LASERTECHNIK GmbH, Wiedner Hauptstrasse 76, A-1040 Vienna, Austria) which was focalized through an UV transparent viewport onto the sample (spot radius about $10 \mathrm{~mm}$ ). During all sample preparation, the UHV pressure was always $<2 \times 10^{-10}$ mbar, we have no indication that the dimaleimide molecules degrade or polymerize in the quartz crucible during the multiple evaporation cycles made for these experiments.

\section{Noncontact Atomic Force Microscopy measurements}

All measurements were performed by a variable-temperature noncontact Atomic Force Microscope (VT-AFM: Scienta Omicron GmbH, Limburger Strasse 75, D - 65232 Taunusstein) operated at room temperature and under UHV conditions ( $<10^{-10} \mathrm{mbar}$ ). The instrument is equipped with home-build in situ and ex situ ncAFM preamplifiers and controlled by an RHK R9 controller (RHK technology, 1050 E. Maple Road, Troy, MI 48083, USA). Careful calibration of the scanner were made by atomic resolution images on clean ionic substrates. ncAFM images were evaluated by means of the WSxM software. ${ }^{67}$ Nanosensor cantilevers were used (PPP$\mathrm{NCL}$ with resonance frequency $\mathrm{f}_{0} \sim 150 \mathrm{kHz}$, spring constant $\mathrm{k} \sim 30 \mathrm{~N} / \mathrm{m}$, quality factor $\mathrm{Q} \sim$ 40000 , and oscillation amplitudes $A_{0}$ of $2-5 \mathrm{~nm}$ ). 


\section{Computational Methods}

Calculations were performed at the PBE0-D3 dispersion-corrected density functional ${ }^{68}$, 69 level of theory within the CP2K software package. ${ }^{70}$ We used high-quality TZV2P molecularly optimized ${ }^{71}$ basis sets for the molecule and DZVP for the $\mathrm{KCl}$ surface, with accompanying GTH pseudopotentials. ${ }^{72}$ A 600 Ry cutoff auxiliary plane-wave basis set ${ }^{73}$ was used and pFIT3 auxiliary Gaussian basis sets used with Auxiliary Density Matrix Method approximation to make the hybrid calculations feasible. Geometry optimization was performed until all forces on atoms were smaller than $0.25 \mathrm{eV} / \mathrm{nm}$. We used a $4 \times 4 \times 2$ conventional unit cell structure for calculations of isolated monomers, and $4 \times 8 \times 2$ supercells for all multimer and transition state calculations. A $4 \times 7 \times 2$ cell was used for the polymer chain calculations. The experimental lattice constant of $\mathrm{KCl}$ was used for convenient comparison to experiment.

Barriers were calculated using Climbing Image Nudged Elastic Band method at PBE0 level of theory with 7 images (5 intermediate images between reactant and product). NEBs were considered adequately converged when the TS energy changed by less than $1 \mathrm{meV}$ in two successive steps. ${ }^{74,75}$

\section{Data Availability Statement}

The datasets generated and analysed during the current study are available within the Article and its Supplementary Information, and/or from the corresponding author upon reasonable request.

\section{Acknowledgements}

The authors acknowledge the financial support from the French National Research Agency through contracts ORGANI'SO (ANR-15-CE09-0017) and PhotoNet (ANR-16-JTIC-0002). Via our membership of the UK's HEC Materials Chemistry Consortium, which is funded by EPSRC (EP/L000202), this work used the ARCHER UK National Supercomputing Service (http://www.archer.ac.uk). S. Clair (IM2NP), F. Palmino (FEMTO-ST), C.M. Thomas (Chimie ParisTech, PSL Research University), and A. L. Shluger (Department of Physics and Astronomy, University College London) are acknowledged for helpful discussion.

\section{Author contributions}

All authors contributed to the scientific discussion and the writing of the manuscript, C. L., L. N. \& and F. P. performed ncAFM experiments, M. F. \& F. C. purified the molecules, and M. W., D. Z. G. \& F. F. C. performed DFT and MD calculations.

\section{Materials \& Correspondence}

Corresponding author:

*Christian.Loppacher@im2np.fr 



\section{Figure Captions:}

Figure 1: Calculated structure and charge-difference plot of a dimaleimide molecule (light blue, $\mathrm{C}$; dark blue, $\mathrm{N}$; red, $\mathrm{O}$; white, $\mathrm{H}$ ) adsorbed on the $\mathrm{KCl}(001)$ surface (light grey, $\mathrm{K}$ and green, $\mathrm{Cl}$ atoms). The molecule's oxygen atoms interact with the potassium cations of the substrate. a) top-view, b) side-view, and c) front-view. Charge density differences are represented in transparent blue (increase) and red (decrease) and show isosurfaces of 0.001 e/Å3 charge difference.

Figure 2: Evaluation of the polymer structure obtained upon polymerization without light. a) Large scale ncAFM image of an as prepared surface with a $1 \mu \mathrm{m}$ long fibre (scan window turned by $45^{\circ}$ with respect to all other images); b) close up showing the typical zig-zag structure of fibres illustrated by four circles overlaid on the fibre c) high-resolution image showing both the substrate lattice constant as well as the zip-arrangement of the polymer fibre (zig-zag structure and periodic cell overlaid); the zig-zag structure reflects a topological rumpling of the fibre due to a coincidence of every fifth molecule with the forth cation along the substrate's crystal axis ( $\mathrm{K}_{\mathrm{kcl}}$, $b_{\mathrm{KCl}}$ ); the rectangle in blue shows the periodic cell used in calculations.d) DFT relaxed structure of the polymer fibre on the $\mathrm{KCl}$ surface.

Figure 3: Influence of weak UV illumination on the growth of the polymer fibres. a,b, ncAFM images of $0.7 \mathrm{ML}$ of dimaleimide deposited onto $\mathrm{KCl}$ without (a) and with UV light (14 hours illumination, deposition during the first minute only) (b).

Figure 4: Proof of the mechanical stability of the fibres. Successful mechanical manipulation was induced by approaching the tip to the part of the fibre indicated by a cross in the top panels. $a, b$, When the upper end of the fibre was manipulated (a), the lower end detached from the substrate step edge and the upper-end geometry changed (b). c,d, A complete fibre (c) stuck to the tip was removed from the surface (d). e,f, The geometry of an already bent fibre (e) was changed (f). 


\section{References}

${ }^{1}$ Kosterlitz, J. M. Nobel Lecture: Topological Defects and Phase Transition. Rev. Mod. Phys. 89, 040501 (2017).

2 Haldane, F. D. M. Nobel Lecture: Topological Quantum Matter. Rev. Mod. Phys. 89, 040502 (2017).

${ }^{3}$ Copie, G. et al. Surface-Induced Optimal Packing of Two-Dimensional Molecular Networks, Phys. Rev. Lett. 114, 066101 (2015).

${ }^{4}$ Ward, M. D. Soft Crystals in Flatland: Unraveling Epitaxial Growth. ACS Nano 10, $6424-6428$ (2016).

${ }^{5}$ Koudia, M., Nardi, E., Siri, O. \& Abel, M. On-surface synthesis of covalent coordination polymers on micrometer scale. Nano Research 10, 933 - 940 (2017).

${ }^{6}$ Klappenberger, F. et al. On-Surface Synthesis of Carbon-Based Scaffolds and Nanomaterials Using Terminal Alkynes. Acc. Chem. Res. 48, 2140 - 2150 (2015).

7 Hla, S. W., Bartels, L., Meyer, G. \& Rieder, K.H. Inducing All Steps of a Chemical Reaction with the Scanning Tunneling Microscope Tip: Towards Single Molecule Engineering. Phys. Rev. Lett. 85, 2777 2780 (2000).

${ }^{8}$ Grill, L. et al. Nano-architectures by covalent assembly of molecular building blocks. Nat. Nanotechnol. 2, $687-691$ (2007).

${ }^{9}$ Lipton-Duffin, J. A., Ivasenko, O., Perepichka, D. F. \& Rosei, F. Synthesis of Polyphenylene Molecular Wires by Surface-Confined Polymerization. Small 5, 592 - 597 (2009).

${ }^{10}$ Lafferentz L. et al. Conductance of a single conjugated polymer as a continuous function of its length. Science 323, 1193 - 1197 (2009).

11 Cai, J. M. et al. Atomically Precise Bottom-up Fabrication of Graphene Nanoribbons. Nature 466, 470 473 (2012).

12 Basagni, A. et al. On-surface photo-dissociation of $\mathrm{C}-\mathrm{Br}$ bonds: towards room temperature Ullmann coupling. Chem. Commun. 51, 12593 - 12596 (2015).

${ }^{13}$ Zhang, Y. Q. et al. Homo-coupling of terminal alkynes on a noble metal surface, Nat. Commun. 3, 1286 -1294 (2012).

14 Gao, H.Y. et al. Glaser coupling at metal surfaces. Angew. Chem. Int. Ed. Engl. 52, $4024-4028$ (2013).

${ }^{15}$ Weigelt, S. et al. Covalent Interlinking of an Aldehyde and an Amine on a Au(111) Surface in Ultrahigh Vacuum. Angew. Chem. Int. Ed. 46, 9227 -9230 (2007).

${ }^{16}$ Zwaneveld, N. et al. Organized Formation of 2D Extended Covalent Organic Frameworks at Surfaces. J. Am. Chem. Soc. 130, $6678-6679$ (2008).

17 Dienstmaier, J. F. et al. Isoreticular Two-Dimensional Covalent Organic Frameworks Synthesized by On-Surface Condensation of Diboronic Acids. ACS Nano 6, 7234 - 7242 (2012).

${ }^{18}$ Clair, S., Abel, M. \& Porte, L. Growth of boronic acid based two-dimensional covalent networks on a metal surface under ultrahigh vacuum. Chem. Commun. 50, 9627 - 9635 (2014).

19 Perepichka, D. F. \& Rosei, F. Extending Polymer Conjugation into the Second Dimension. Science 323, $216-217$ (2009).

${ }^{20}$ Franc, G. \& Gourdon, A. Covalent networks through on-surface chemistry in ultra-high vacuum: state-of-the-art and recent developments. Phys. Chem. Chem. Phys. 13, $14283-14292$ (2011).

${ }^{21}$ Fan, Q., Gottfried, J.M. \& Zhu, J. Surface-Catalyzed C-C Covalent Coupling Strategies toward the Synthesis of Low-Dimensional Carbon-Based Nanostructures. Acc. Chem. Res. 48, 2484 - 2494 (2015). 22 Payamyar, P., King, B. T., Öttinger, H. C. \& Schlüter, A.D. Two-dimensional polymers: concepts and perspectives, Chem. Commun. 52, 18 - 34 (2016).

${ }^{23}$ Wakayama, Y. On-surface molecular nanoarchitectonics: From self-assembly to directed assembly. Jap. J. Appl. Physics 55, 1102AA (2016).

24 Di Giovannantonio, M. et al. Mechanistic Picture and Kinetic Analysis of Surface-Confined Ullmann Polymerization. J. Am. Chem. Soc. 138, 16696 - 16702 (2016).

${ }^{25}$ Cai, Y. H., Shao, Y. X. \& Xu, G. Q. Photoinduced Construction of a Second Covalently Bonded Organic Layer on the Si(111)-7 x 7 Surface. J. Am. Chem. Soc. 129, $8404-8405$ (2007). 
26 Deshpande, A. et al. Self-Assembly and Photopolymerization of Sub-2 nm One-Dimensional Organic Nanostructures on Graphene. J. Am. Chem. Soc. 134, 16759 - 16764 (2012).

27 Gao, H.-Y. et al. Photochemical Glaser Coupling at Metal Surfaces. J. Phys. Chem. C 118, $6272-$ 6277 (2014).

28 Shen, Q. et al. Self-assembled two-dimensional nanoporous molecular arrays and photoinduced polymerization of 4-bromo-4'-hydroxybiphenyl on Ag(111). J. Chem. Phys. 142, 101902 (2015).

29 Okawa, Y. \& Aono, M. Nanoscale control of chain polymerization. Nature 409, $683-684$ (2001).

${ }^{30}$ Clair, S., Ourdjini, O., Abel, M. \& Porte, L. Tip- or electron beam-induced surface polymerization. Chem. Commun. 47, $8028-8030$ (2011).

31 Dinca, L. E. et al. Tip-induced C-H activation and oligomerization of thienoanthracenes. Chem. Commun. 50, $8791-8793$ (2014).

32 Qiu, X. H., Nazin, G. V. \& Ho, W. Vibrationally Resolved Fluorescence Excited with Submolecular Precision. Science 299, $542-546$ (2003).

33 Bieri, M. et al. J. Am. Chem. Soc. 132, $16669-16676$ (2010).

$34 \mathrm{Gao}, \mathrm{H} .-\mathrm{Y}$. et al. Decarboxylative Polymerization of 2,6-Naphthalenedicarboxylic Acid at Surfaces. J. Am. Chem. Soc. 136, 9658 - 9663 (2014).

35 Bombis, C. et al. Single Molecular Wires Connecting Metallic and Insulating Surface Areas. Angew. Chem. Int. Ed. 48, 9966 - 9970 (2009).

${ }^{36}$ Abel, M., Clair, S., Ourdjini, O., Mossoyan, M. \& Porte, L. Single Layer of Polymeric Fe-Phthalocyanine: An Organometallic Sheet on Metal and Thin Insulating Film. J. Am. Chem. Soc. 133, 1203 - 1205 (2011).

${ }^{37}$ Dienel, T. et al. Dehalogenation and Coupling of a Polycyclic Hydrocarbon on an Atomically Thin Insulator. ACS Nano 8, 6571 - 6579 (2014).

${ }_{38}$ Zhao, W., Dong, L., Huang, C., Win, Z. M. \& Lin, N. Cu- and Pd-catalyzed Ullmann reaction on a hexagonal boron nitride layer. Chem. Commun. 52, 13225 (2016).

${ }^{39}$ Steurer, W., Fatayer, S., Gross, L. \& Meyer, G. Probe-based measurement of lateral single-electron transfer between individual molecules. Nature Comm. 6, 8353 - 8358 (2015).

40 Kittelmann, M. et al. On-Surface Covalent Linking of Organic Building Blocks on a Bulk Insulator. ACS Nano 5, 8420 - 8425 (2011).

${ }^{41}$ Kittelmann, M., Nimmrich, M., Lindner, R., Gourdon, A. \& Kühnle, A. Sequential and Site-Specific OnSurface Synthesis on a Bulk Insulator. ACS Nano 7, 5614 - 5620 (2013).

42 Lindner, R. et al. Substrate Templating Guides the Photoinduced Reaction of C60 on Calcite. Angew. Chem. Int. Ed. 53, 7952 - 7955 (2014).

${ }^{43}$ Guo, C. et al. Mechanisms of Covalent Dimerization on a Bulk Insulating Surface. J. Phys. Chem. C 121, 10053 - 10062 (2017).

${ }^{44}$ Richter, A. et al. Diacetylene polymerization on a bulk insulator surface. Phys. Chem. Chem. Phys. 19, $15172-15176$ (2017).

45 Palma,C.-A. et al. Photoinduced C-C Reactions on Insulators toward Photolithography of Graphene Nanoarchitectures. J. Am. Chem. Soc. 136, 4651 - 4658 (2014).

${ }^{46}$ Rahe, P. et al. Tuning Molecular Self-Assembly on Bulk Insulator Surfaces by Anchoring of the Organic Building Blocks. Adv. Mater. 25, 3948 - 3956 (2013).

47 Zhong, D. et al. Linear Alkane Polymerization on a Gold Surface. Science 334, 213 - 216 (2011).

48 Champness, N.R. Making the right connections. Nature Chem. 4, $149-150$ (2012).

49 Díaz Arado, O. et al. On-Surface Azide-Alkyne Cycloaddition on Au(111). ACS Nano 7, 8509 - 8515 (2013).

50 Chen, Q. et al. Steering On-Surface Reactions by a Self-Assembly Approach. Angew. Chem. Int. Ed. $56,5026-5030$ (2017).

51 Sedona, F. et al. Fullerene/Porphyrin Multicomponent Nanostructures on $\mathrm{Ag}(110)$ : From Supramolecular Self-Assembly to Extended Copolymers. ACS Nano 4, 5147 - 5154 (2010).

52 Guan, C. -Z., Wang, D. \& Wan, L. -J. Construction and repair of highly ordered 2D covalent networks by chemical equilibrium regulation. Chem. Commun. 48, $2943-2945$ (2012).

53 Webster, O. W. Living Polymerization Methods. Science 251, 887 - 893 (1991).

${ }^{54}$ Zakir Hossain, Md., Kato, H.S. \& Kawai, M. Controlled Fabrication of 1D Molecular Lines Across the Dimer Rows on the Si(100)-(2 x 1)-H Surface through the Radical Chain Reaction. J. Am. Chem. Soc. 127, $15030-15031$ (2005). 
55 Okawa, Y., Akai-Kasaya, M., Kuwahara, Y., Mandala, S. K. \& Aono, M. Controlled chain polymerisation and chemical soldering for single-molecule electronics. Nanoscale 4, $3013-3028$ (2012).

56 Sun, Q. et al. On-Surface Formation of One-Dimensional Polyphenylene through Bergman Cyclisation. J. Am. Chem. Soc. 135, $8448-8451$ (2013).

57 Riss, A. et al. Local Electronic and Chemical Structure of Oligo-acetylene Derivatives Formed Through Radical Cyclizations at a Surface. Nanolett. 14, 2251 - 2255 (2014).

58 Verstraete, V., Hirsche, B. E., Greenwood, J. \& De Feyter, S. Confined Polydiacetylene Polymerization Reactions for Programmed Length Control. Chem. Commun. 53, 4207 - 4210 (2017).

59 Dolci, E. et al. Maleimides as a Building Block for the Synthesis of High Performance Polymers. Polymer Reviews 56, 512 - 556 (2016).

60 Tsurkan, M. V., Jungnickel, C., Schlierf, M. \& Werner, C. Forbidden Chemistry: Two-Photon Pathway in [2+2] Cycloaddition of Maleimides. J. Am. Chem. Soc. 139, $10184-10187$ (2017).

61 Hall, D. J., Van Den Berghe, H. M. \& Dove A. P. Synthesis and Post-Polymerization Modification of Maleimide-Containing Polymers By 'Thiol-Ene' Click and Diels-Alder Chemistries. Polymer International 60, $1149-1157$ (2011).

62 Matsumoto, A., Kubota, T., \& Ostsu T. Radical Polymerization of N-(AlkylSubsitutedphenyl)maleimides: Syntheis of Thermally Stable Polymers Soluble in Nonpolar Solvents. Macromol. 23, 4508-4513 (1990).

${ }^{63}$ Stille, J. K. Step-Growth Polymerization. J. Chem. Educ. 58, 862 - 866 (1981).

64 Vazquez, C. P., Joly-Duhamel, C. \& Boutevin, B. Photopolymerization Without Photoinitiator of Bismaleimide-Containing Oligo(Oxypropylene)s: Effect of Oligoethers Chain Length. Macromol. Chem. Phys. 210, $269-278$ (2009).

65 Amrous, A. et al. Design and Control Over the Morphology of Self-Assembled Films on Ionic Substrates. Adv. Mater. Interfaces 1400414 (2014).

${ }^{66}$ Matena, M., Riehm, T., Stöhr, M., Jung, T.A. \& Gade, L.H. Transforming Surface Coordination Polymers into Covalent Surface Polymers: Linked Polycondensed Aromatics through Oligomerization of $\mathrm{N}$-Heterocyclic Carbene Intermediates. Angew. Chem. Int. Ed. 47, 2414 - 2417 (2008).

67 Horcas, I. et al. WSXM: a software for scanning probe microscopy and a tool for nanotechnology. Rev. Sci. Instrum. 78, 013705 (2007); www.wsxmsolutions.com

68 Perdew, J. P., Burke, K. \& Ernzerhof, M. Generalized gradient approximation made simple. Phys. Rev. Lett. 77, 3865 - 3868 (1996).

${ }^{69}$ Grimme, S. Density functional theory with London dispersion corrections. WIREs Comput. Mol. Sci. 1, $211-228(2011)$.

70 Hutter, J., lannuzzi, M., Schiffmann. F. \& VandeVondele, J. cp2k: atomistic simulations of condensed matter systems. WIREs Comput. Mol. Sci. 4, 15 - 25 (2014).

${ }^{71}$ VandeVondele, J. \& Hutter, J. Gaussian basis sets for accurate calculations on molecular systems in gas and condensed phases. J. Chem. Phys. 127, 114105 (2007).

72 Krack, M. Pseudopotentials for $\mathrm{H}$ to $\mathrm{Kr}$ optimized for gradient-corrected exchange-correlation functionals. Theor. Chem. Acc. 114, 145 - 152 (2005).

${ }^{73}$ VandeVondele, J. et al. QUICKSTEP: Fast and accurate density functional calculations using a mixed Gaussian and plane waves approach. Comput. Phys. Commun. 167, $103-128$ (2005).

${ }^{74}$ Henkelman, G., Uberuaga, B. P. \& Jonsson, H. A climbing image nudged elastic band method for finding saddle points and minimum energy paths. J. Chem. Phys. 113, $9901-9904$ (2000).

75 Henkelman, G. \& Jonsson, H. Improved tangent estimate in the nudged elastic band method for finding minimum energy paths and saddle points. J. Chem. Phys. 113, $9978-9985$ (2000). 
Figure Files:

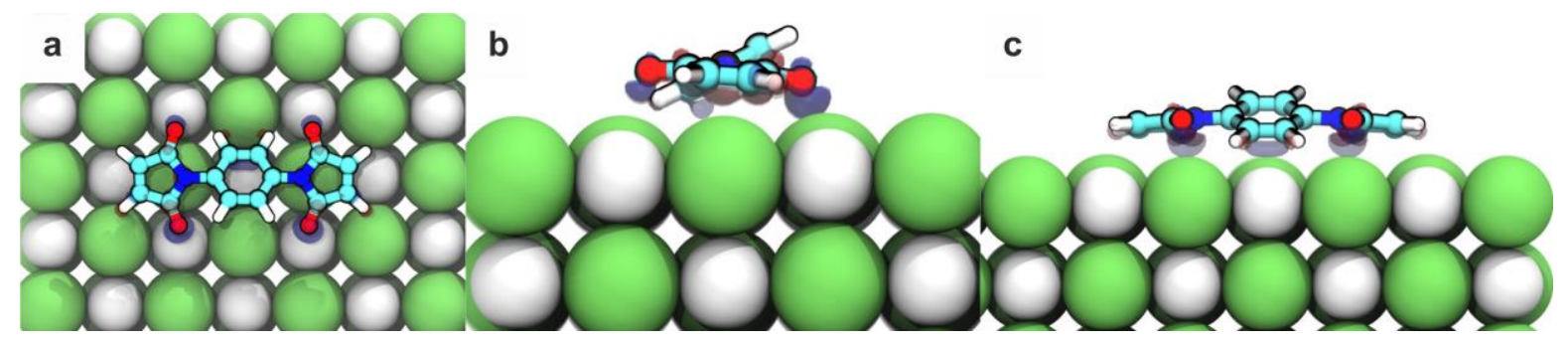

Figure 1: Calculated structure and charge-difference plot of a dimaleimide molecule adsorbed on the $\mathrm{KCl}(001)$ surface (white $\mathrm{K}$ and green $\mathrm{Cl}$ atoms). The molecule's oxygen atoms interact with the potassium cations of the substrate. a) top-view, b) side-view, and c) front-view. Charge density differences are in transparent blue (increase) and red (decrease) and show isosurfaces of 0.001 e/Å3 charge difference. 


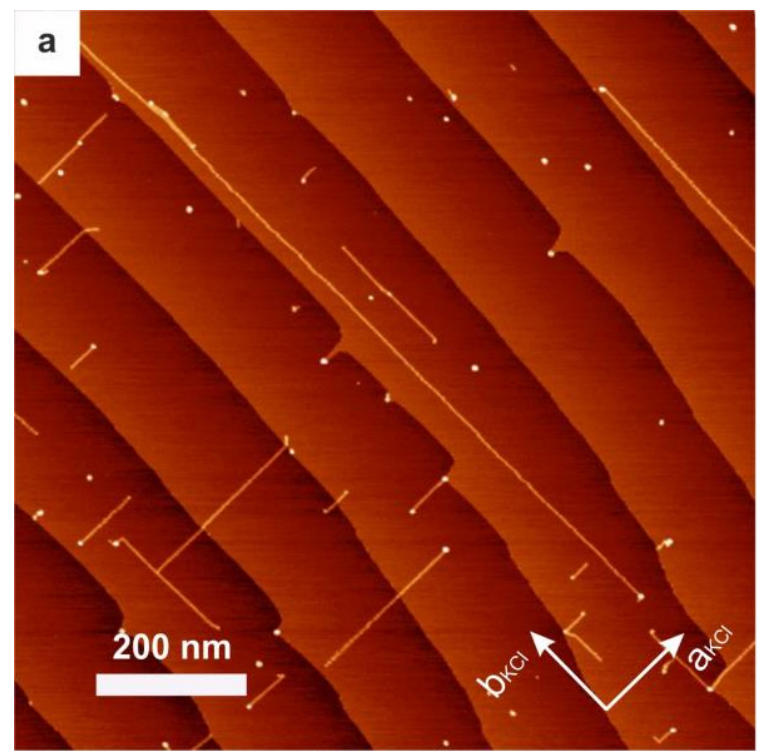

$\Delta \mathrm{z}(\mathrm{nm})$

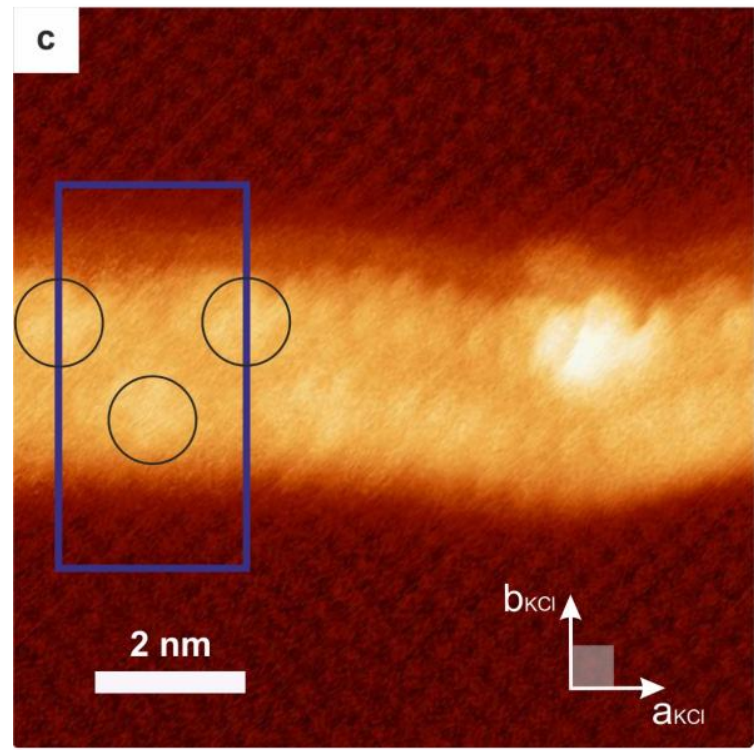

$\Delta \mathrm{z}(\mathrm{nm})$

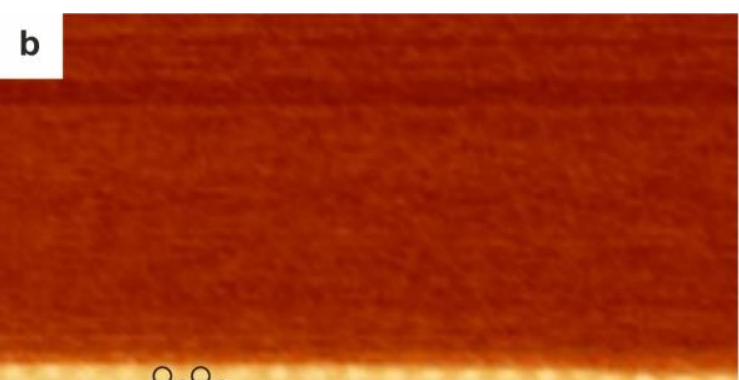

$\mathrm{O}^{\circ} \mathrm{O}$

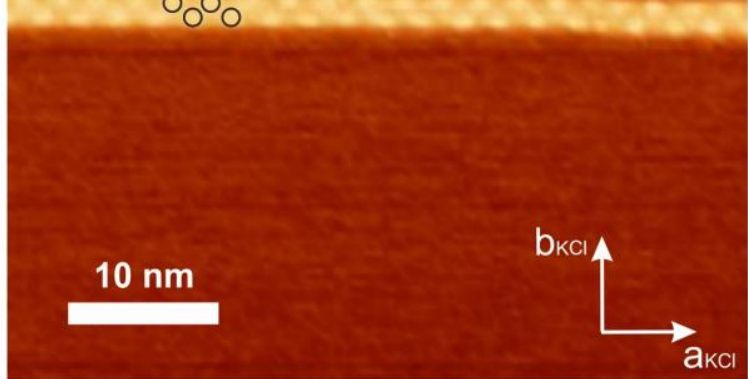

$\Delta \mathbf{z}(\mathrm{nm})$ 1.0

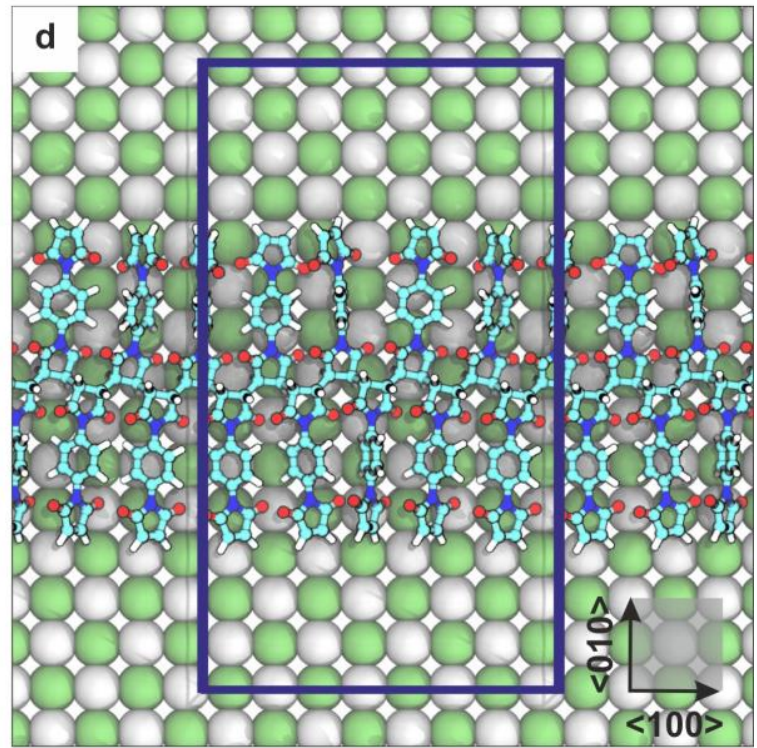

Figure 2: Evaluation of the polymer structure obtained upon polymerization without light. a) Large scale ncAFM image of an as prepared surface with a $1 \mu \mathrm{m}$ long fibre (scan window turned by $45^{\circ}$ with respect to all other images); b) close up showing the typical zig-zag structure of fibres; c) high-resolution image showing both the substrate lattice constant as well as the zip-arrangement of the polymer fibre (zig-zag structure and periodic cell overlaid); the zig-zag structure reflects a topological rumpling of the fibre due to a coincidence of every fifth molecule with the forth cation along the substrate's crystal axis $\left(a_{k C l}, b_{K c l}\right)$; d) DFT relaxed structure of the polymer fibre on the $\mathrm{KCl}$ surface (white $\mathrm{K}$ and green $\mathrm{Cl}$ atoms). The box drawn is the periodic cell used in calculations. 


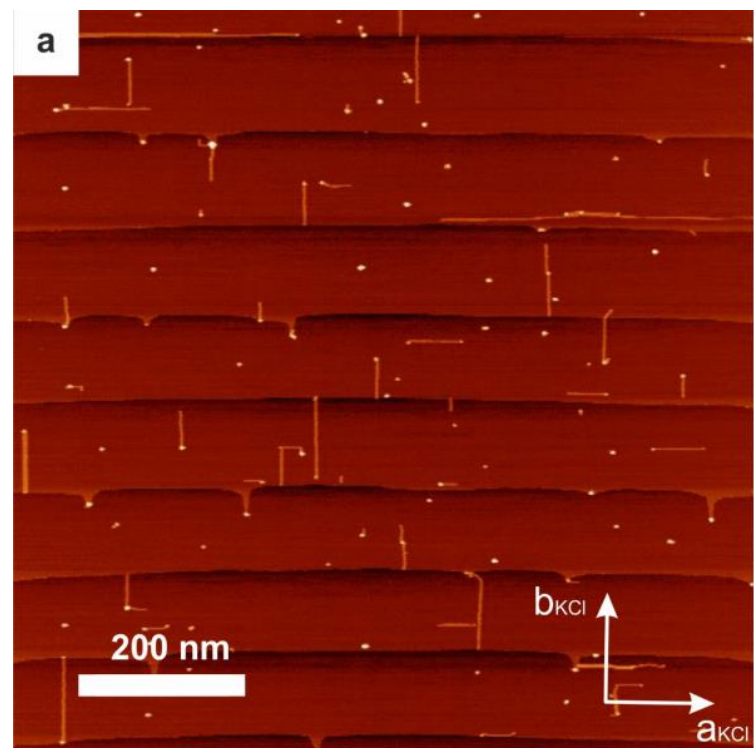

$\Delta \mathbf{z}(\mathrm{nm})$

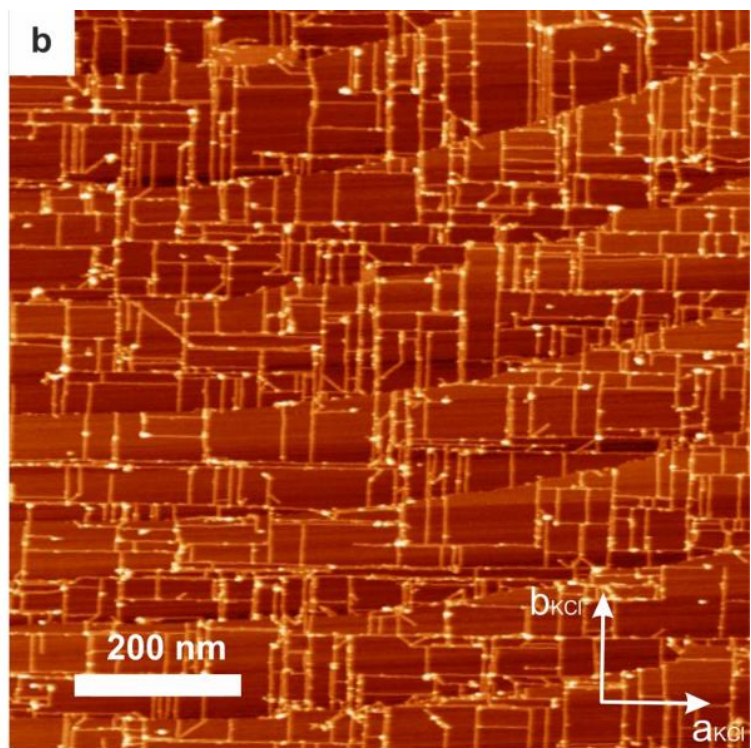

$\Delta \mathbf{z}(\mathrm{nm})$ 2.0

Figure 3: Influence of weak UV illumination illumination on the growth of the polymer fibres. ncAFM images of $0.7 \mathrm{ML}$ of dimaleimide deposited onto $\mathrm{KCl}$ without (a) and with (b) UV light (14 hours illumination, deposition during the first minute only). 


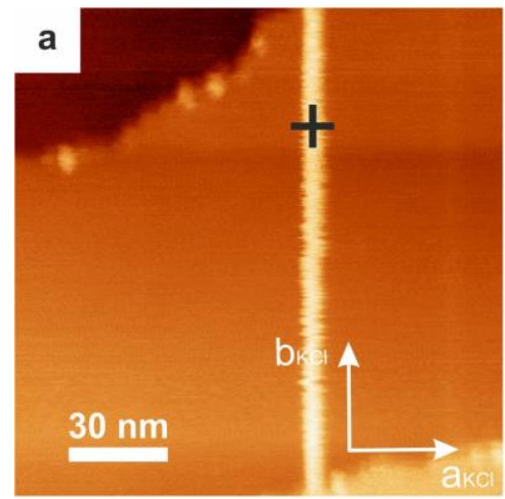

$\Delta \mathrm{z}(\mathrm{nm}) 0 \square 0.8$

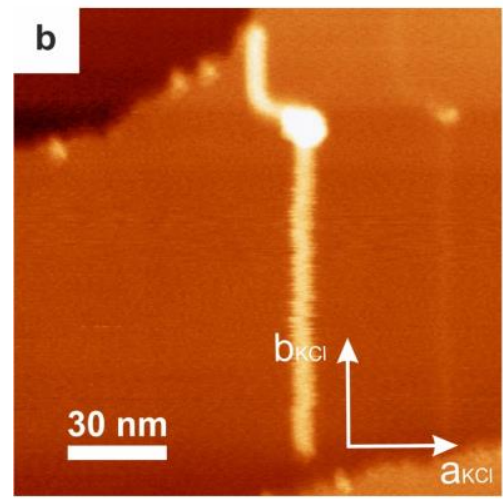

$\Delta \mathrm{z}(\mathrm{nm}) 0 \square 0.8$

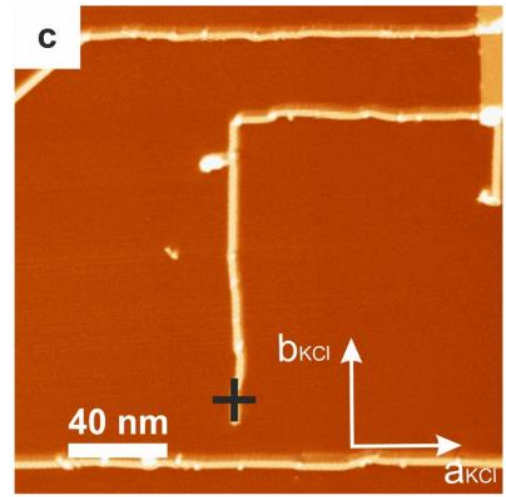

$\Delta \mathrm{z}(\mathrm{nm}) \mathbf{0} 1.0$

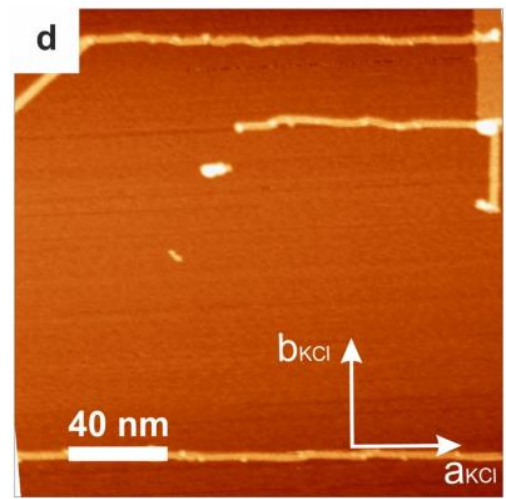

$\Delta \mathrm{z}(\mathrm{nm}) 0 \square 1.0$

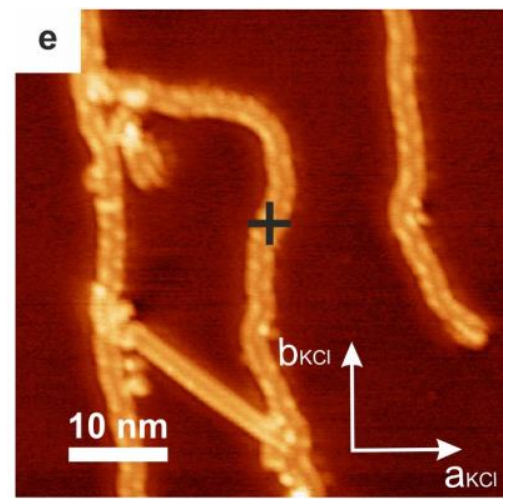

$\Delta \mathrm{z}(\mathrm{nm}) \mathbf{0} \square 1.1$

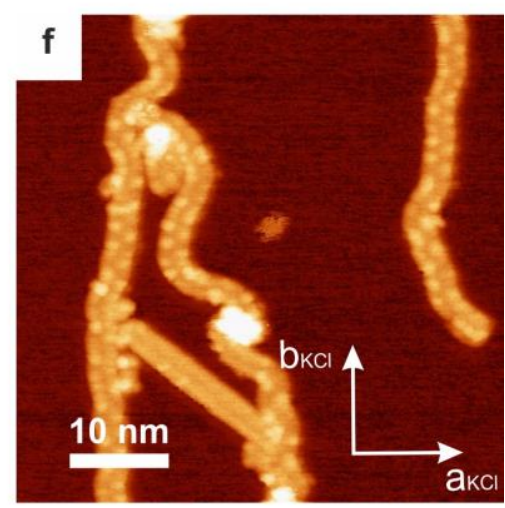

$\Delta \mathrm{z}(\mathrm{nm}) \mathbf{0} \square 1.1$

Figure 4: Proof of the mechanical stability by means of three different examples of a successful mechanical manipulation induced by approaching the tip to the part of the fibre indicated by a cross in figures a, c, and e. (images a \& b) The upper end of the fibre was manipulated $\rightarrow$ the lower end detached from the substrate step edge and the upper-end geometry changed; ( $c \& d$ ) a complete fibre stuck to the tip and was removed from the surface; $(e \& f)$ the geometry of an already bent fibre was changed. 